\title{
Data-Driven Identification of Adverse Event Reporting Patterns for Japan in VigiBase, the WHO Global Database of Individual Case Safety Reports
}

\author{
Rika Wakao $^{1} \cdot$ Henric Taavola ${ }^{2}\left(\right.$ Hovisa Sandberg $^{2} \cdot$ Eiko Iwasa $^{1} \cdot$ Saori Soejima ${ }^{1} \cdot$ Rebecca Chandler $^{2}$. \\ G. Niklas Norén ${ }^{2}(1)$
}

Published online: 26 September 2019

(c) The Author(s) 2019

\begin{abstract}
Introduction Adverse event reporting patterns vary between countries, reflecting differences in reporting culture, clinical practice and underlying patient populations. Japan collects about 60,000 domestic adverse event reports yearly and shares serious reports with the World Health Organization (WHO) Programme for International Drug Monitoring in VigiBase, the WHO global database of individual case safety reports. Understanding these reports in the global context can be helpful for regulators worldwide and can aid hypothesis-generation for Japanese-specific vulnerabilities to adverse drug reactions. Objective The objective of this study was to explore differences in the reporting of adverse events between Japan and other countries.

Methods vigiPoint is a method for data-driven exploration in pharmacovigilance. It outlines data subsets, pinpoints key features and facilitates expert review, using odds ratios subjected to statistical shrinkage to distinguish one data subset from another. Here, we compared 260,000 Japanese reports in E2B format classified as serious and received in VigiBase between 2013 and 2018 with 2.5 million reports from the rest of the world (of which 51\% are from the USA). Reporting patterns for which the $99 \%$ credibility interval of the shrunk log-odds ratios were above 0.5 or below -0.5 were flagged as key features. The shrinkage was set to the vigiPoint default corresponding to $1 \%$ of the size of the Japanese data subset. As a sensitivity analysis, additional vigiPoint comparisons were performed between Japan and, in turn, Africa, the Americas, the Americas except the USA and Canada, Asia and Europe.

Results There were higher reporting rates in Japan from physicians (83\% vs. 39\%) and pharmacists (17\% vs. 10\%). It was also more common to see reports with more than five drugs per report (22\% vs. $14 \%$ ) and with a single adverse event $(72 \%$ vs. $45 \%$ ). More than half of the Japanese reports had a vigiGrade completeness score above 0.8 compared with about one in five from the rest of the world. There were more reports than expected for patients aged 70-89 years and fewer reports for adults aged 20-59 years. Adverse events reported more often in Japan included interstitial lung disease, abnormal hepatic function, decreased platelet count, decreased neutrophil count and drug eruption. Adverse events reported less often included death, fatigue, dyspnoea, pain and headache. Drugs reported more often in Japan included prednisolone, methotrexate and peginterferon alfa- $2 \mathrm{~b}$. Drugs reported less often included rosiglitazone and adalimumab as well as blood substitutes and perfusion solutions. The findings were generally robust to the sensitivity analysis except for the less often reported drugs, many of which were rarely reported in most countries, except in the USA.

Conclusion Analysis of Japanese adverse event reporting patterns in a global context has revealed key features that may reflect possible pharmaco-ethnic vulnerabilities in the Japanese, as well as differences in adverse event reporting and clinical practice. This knowledge is essential in the global collaboration of signal detection afforded by the WHO Programme for International Drug Monitoring.
\end{abstract}

Electronic supplementary material The online version of this article (https://doi.org/10.1007/s40264-019-00861-y) contains supplementary material, which is available to authorized users.

Extended author information available on the last page of the article

\section{Background}

Japan joined the World Health Organization (WHO) Programme for International Drug Monitoring in 1972. At the time, it was one of only 14 member countries and the 


\section{Key Points}

Analysis of Japanese adverse event reporting patterns in a global context has revealed key features that may reflect possible pharmaco-ethnic vulnerabilities in the Japanese, as well as differences in adverse event reporting and clinical practice.

Adverse events reported more often in Japan included interstitial lung disease, abnormal hepatic function, decreased platelet count, decreased neutrophil count and drug eruption.

More reports from Japan had high completeness, were submitted by physicians and included a single adverse event term.

programme's sole Asian representative. By February 2019, the WHO programme has grown to 134 full member countries (and 30 associates), including most countries in Asia.

Japan's Pharmaceuticals and Medical Devices Agency (PMDA) was established and came into service on 1 April 2004. Prior to that, the Japanese pharmacovigilance centre was based in a bureau of the former Ministry of Health and Welfare. Domestic adverse drug reaction (ADR) monitoring was initiated in Japan in 1967, and the PMDA safety department took over the collection of spontaneous reports in 2004. The PMDA focuses on three key service areas: relief services for persons suffering adverse health effects related to the use of medical products; medical product regulatory reviews; and drug safety measures. The PMDA's safety department functions as the National Pharmacovigilance Centre of the WHO Programme for International Drug Monitoring. The PMDA collects around 60,000 domestic reports of adverse events every year. Reports of adverse events are shared within the international pharmacovigilance community through VigiBase, the WHO global database of individual case safety reports. In total, there are nearly 400,000 reports from Japan in VigiBase, with a current growth of about 60,000 new reports shared internationally every year (February 2019).

Adverse event reporting patterns are expected to vary between countries due to differences in the underlying populations and clinical settings as well as in reporting cultures and regulation. A better understanding of these differences may point to strengths and areas of opportunity for a country's pharmacovigilance system. It may also identify susceptibilities to certain types of ADRs in a country, something that is more difficult in a single country's database where there may be limited or no international comparisons available.

\section{Aims}

The objective of this study was to explore whether the reporting of serious suspected ADRs differs in important ways between Japan and other countries in the WHO Programme for International Drug Monitoring and to discuss possible explanations for such differences.

\section{Methods}

\subsection{VigiBase}

VigiBase is the WHO global database of individual case safety reports. Reports are collected and shared by the 134 (February 2019) members of the WHO Programme for International Drug Monitoring. The VigiBase data used in this study is from 2 January 2018 and contains 16.3 million reports, of which around 330,000 are from Japan, not counting suspected duplicate reports. Reports in VigiBase encode drugs using WHODrug and adverse events using the Medical Dictionary for Regulatory Activities (MedDRA ${ }^{\circledR}$ ). ${ }^{1}$ MedDRA ${ }^{\circledR}$ is a hierarchical terminology with five levels: Lowest Level Terms (LLTs), Preferred Terms (PTs), High Level Terms (HLTs), High Level Group Terms (HLGTs) and System Organ Classes (SOCs). Beyond that, there are Standardised MedDRA Queries (SMQs), which are pre-defined sets of MedDRA ${ }^{\circledR}$ terms aimed at effective case retrieval for important pharmacovigilance topics; the latter exists in both narrow and broad versions.

To reduce the impact of some potential confounders when comparing reports from Japan with reports from the rest of the world (RoW), we employed the following exclusion criteria:

- Suspected duplicate reports: as identified with vigiMatch [1].

- Reports received before 2013: to reflect more recent reporting patterns and the current composition of the WHO Programme for International Drug Monitoring.

- Reports not in the E2B format: applied since all reports shared by the PMDA in this time period are in the E2B format, and since older reporting formats such as INTDIS (International Drug Information System) do not provide corresponding markers of seriousness and have limitations on the number of listed drugs and adverse events per report.

\footnotetext{
${ }^{1}$ MedDRA $^{\circledR}$ terminology is the international medical terminology developed under the auspices of the International Conference on Harmonisation of Technical Requirements for Registration of Pharmaceuticals for Human Use (ICH). The MedDRA ${ }^{\circledR}$ trademark is owned by the International Federation of Pharmaceutical Manufacturers and Associations (IFPMA) on behalf of ICH.
} 
- Reports not marked as serious: as indicated by an E2B Serious field and/or Seriousness criteria fields. Applied since nearly all reports collected by the PMDA as individual case safety reports are serious in nature.

- Reports on medication errors: as defined by a reported term that sorts under the MedDRA HLGT medication errors and other product use errors and issues. Applied since the PMDA does not collect reports on medication errors.

- Reports on lack of effect: as defined by either of the reported MedDRA PTs drug ineffective and drug ineffective for unapproved indication. Applied since the PMDA does not collect individual case safety reports on lack of effect.

\section{2 vigiPoint}

vigiPoint is a method for data-driven exploration in pharmacovigilance, seeking to outline data subsets, pinpoint key features and facilitate expert review. For a data subset of interest (in our case Japan), it contrasts the relative frequency of different covariate values to those in one or more comparator subsets (in our primary analysis, all reports from other countries than Japan) and highlights substantial deviations that have both a certain magnitude and apply to a certain proportion of the data points-not every statistically significant association will be highlighted as a key feature [2].

vigiPoint's basic measure of association is the log-odds ratio. vigiPoint utilises a combination of relatively strong statistical shrinkage, broad credibility intervals and a threshold on magnitude to identify key features that have both high enough relative frequencies to reflect general features of the subset of interest and exhibit large enough differences in magnitude between the subset of interest and the comparator.

For each feature of potential interest, data can be summarized in a standard $2 \times 2$ contingency table as in Table 1 . The shrinkage odds ratio of vigiPoint is considered an observedto-expected ratio and is obtained as the Bayesian posterior mean of an intensity parameter $\mu$ for a Poisson $\operatorname{Po}(\mu \cdot E)$-distributed observed number of reports $(\mathrm{O})$, where the expected value $E$ is $b c / d$. With a Gamma prior distribution with hyperparameter $k$ for $\mu$ : $\mathrm{G}(\mathrm{k} ; \mathrm{k})$, the corresponding posterior distribution for $\mu$ is also Gamma (but with parameters $\mathrm{O}+\mathrm{k}$ and $\mathrm{E}+\mathrm{k})$, and the shrunk odds ratio is computed as follows [3]:

$$
\frac{a+k}{\frac{b c}{d}+k}
$$

Credibility intervals that indicate a range of values of $\mu$ compatible with data can be calculated by the inverse of the Gamma cumulative distribution function. We refer to the lower $C \%$ credibility interval limit of the shrunk log-odds
Table 1 A standard $2 \times 2$ contingency table

\begin{tabular}{lll}
\hline & Feature + & Feature - \\
\hline Subset of interest & $a$ & $b$ \\
Comparator & $c$ & $d$ \\
\hline
\end{tabular}

ratio (or the upper $C \%$ confidence interval limit in the case of negative associations) as the vigiPoint score, where $C$ is the size of the credibility interval. When this exceeds the pre-defined threshold $T$ (or falls below $-T$ for negative association), the corresponding covariate value or range is highlighted as a key feature. For transparency, odds ratios presented as part of the results are unshrunk.

Throughout this paper, we use the standard implementation of vigiPoint with $C=99, k=0.01 \times n$ (where $n$ is the size of the subset of interest; in our case the selected reports from Japan) and $T=0.5[2]$.

\subsection{Study Design}

In our study, Japanese reports in VigiBase are the data subset of interest and reports from the RoW in VigiBase are the primary comparator. The covariates of interest are patient age grouped in 10-year bands, patient sex, adverse events (primarily MedDRA ${ }^{\circledR} \mathrm{PTs}$ ), suspected drugs (primarily WHODrug substance names), proportion of fatal cases, ${ }^{2}$ number of reported adverse event terms per report, number of reported drugs per report (including those marked as concomitant), type of reporter, country of origin and the amount of information per report as measured by the vigiGrade completeness score [4]. For vigiGrade we defined high completeness as a score of 0.8 or higher.

Following up on the initial analysis, we performed a nested vigiPoint analysis of Japanese reports on interstitial lung disease (ILD) in which we used two parallel comparators: Japanese reports of other adverse events and ILD reports from the RoW. This is based on the observation that ILD is identified as the adverse event most highly ranked by vigiPoint for reports from Japan in the primary analysis. The covariates in this nested analysis were the same as in the primary analysis, with the exception of adverse event terms which are excluded, since ILD is used to define the subset of interest.

The RoW is a heterogeneous comparator composed of reports from different regions across the world. What looks

\footnotetext{
${ }^{2}$ A report is considered a fatal case if fields such as "Patient death date", "Patient autopsy" or "Patient death date" are populated with valid values and/or "Seriousness criteria" is set to "Results in death", "Reaction outcome" is set to "Fatal" and/or the reported reaction term is considered a fatal term (such as "Completed suicide", "Death neonatal", "Brain death", "Sudden infant death syndrome", "Sudden death", etc.).
} 
to be differences between Japan and the RoW might in reality be driven by outlying reporting patterns of specific countries or regions included in the RoW. As a sensitivity analysis, we therefore performed additional vigiPoint comparisons between Japan and, in turn, Africa, the Americas, the Americas except the USA and Canada, Asia and Europe. Oceania was not included as a comparator since there were no E2B reports from this region in VigiBase by January 2018.

\section{Results}

After applying the exclusion criteria described in Sect. 3.1, a total of 261,052 Japanese reports and 2,522,856 reports from the RoW remained, as shown in Fig. 1. The exclusion criteria with greatest influence on the comparator are the removal of non-serious reports and the removal of reports from before 2013.

The countries contributing the highest number of reports to the comparator are displayed in Table 2 .

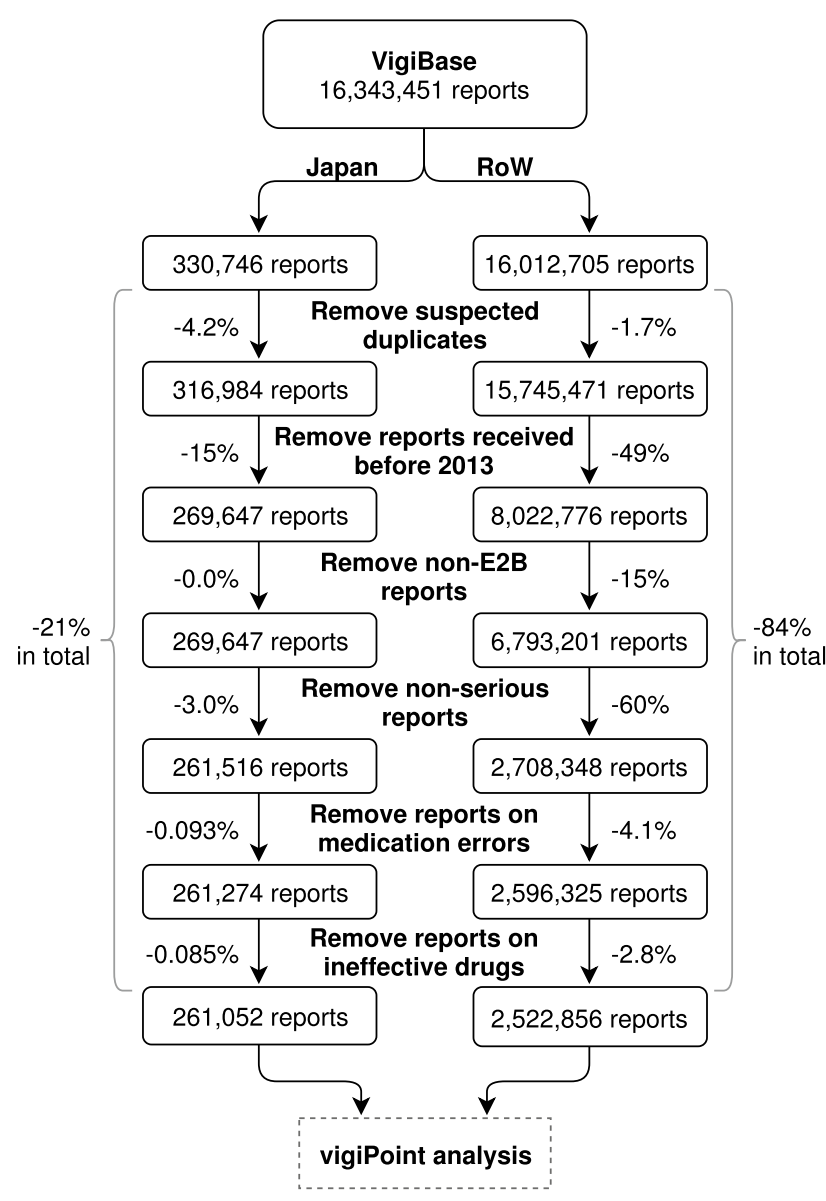

Fig. 1 Flowchart of applied report filters for Japan and the rest of the world (RoW)
Table 2 Top ten contributing countries after application of the exclusion criteria

\begin{tabular}{lr}
\hline Country & Reports $[n(\%)]$ \\
\hline USA & $1,286,362(51)$ \\
France & $222,498(8.8)$ \\
UK & $152,577(6.0)$ \\
Germany & $141,781(5.6)$ \\
Canada & $137,980(5.5)$ \\
Italy & $85,425(3.4)$ \\
Republic of Korea & $70,523(2.8)$ \\
Spain & $55,102(2.2)$ \\
India & $52,203(2.1)$ \\
Netherlands & $34,390(1.4)$ \\
\hline
\end{tabular}

Overall, $77 \%$ of the reports from both Japan and the RoW are spontaneous reports, with $20 \%$ reports from studies for Japan and $21 \%$ for the RoW. For Japan, $49 \%$ of the reports that provide information on patient sex are for women, whereas the corresponding proportion for the RoW is $57 \%$.

\subsection{Key Features of Japanese Reports}

Key features of reports from Japan with higher relative reporting rates compared with the RoW are shown in Table 3 and those with lower relative reporting rates are shown in Table 4. Most reports in the Japanese subset are from physicians, with a substantial contribution also from pharmacists; the relative contributions of these two professional groups are around twice those in reports from the RoW. The proportion of reports with consumer/non-health professional as one of the reporter qualifications on the other hand is only one-quarter of that in reports from the RoW, and other health professional and lawyers are also less frequent reporter qualifications than for the RoW. However, the reporting rate from lawyers in the RoW appears to be driven by reports from the USA, and in our sensitivity analysis, the lower reporting rate from lawyers in Japan was only highlighted as a key feature in the comparison against the Americas.

The distributions of reports by patient age differs between Japan and the RoW, with higher proportions of reports from Japan on patients between 70 and 89 years of age, but lower proportions of reports on adults between 20 and 59 years of age. The proportions of reports for children and adolescents are similar as illustrated by Fig. 2 .

It is more common for reports from Japan to have a single reported adverse event term, and less common to have three or more reported adverse event terms. In contrast, it is less common for reports from Japan to have a single reported drug and more common to have more than five reported drugs. In other words, reports from Japan list on average 
Table 3 Key features with higher relative reporting rates in the Japanese subset

\begin{tabular}{lllll}
\hline Feature & $\begin{array}{l}\text { Japan }(\%) \\
(n=261,052)\end{array}$ & $\begin{array}{l}\text { Rest of the world } \\
(\%)(n=2,522,856)\end{array}$ & Odds ratio & vigiPoint score \\
\hline Reported by: physician & 83 & 39 & 7.5 & 2.8 \\
Completeness: high & 52 & 21 & 4.1 & 1.9 \\
No. of adverse events per report: 1 & 72 & 45 & 3.1 & 1.6 \\
Reported by: pharmacist & 17 & 10 & 1.9 & 0.9 \\
No. of drugs per report: $>5$ & 22 & 14 & 1.8 & 0.8 \\
Patient age: $70-79$ years & 26 & 17 & 1.7 & 0.7 \\
Patient age: $80-89$ years & 15 & 9 & 1.7 & 0.7 \\
\hline
\end{tabular}

Table 4 Key features with lower relative reporting rates in the Japanese subset

\begin{tabular}{lllll}
\hline Feature & $\begin{array}{l}\text { Japan }(\%) \\
(n=261,052)\end{array}$ & $\begin{array}{l}\text { Rest of the world (\%) } \\
(n=2,522,856)\end{array}$ & Odds ratio & vigiPoint score \\
\hline Reported by: consumer/non-health professional & 9.2 & 36 & 0.18 & -2.3 \\
No. of adverse events per report: $>5$ & 1.7 & 11 & 0.15 & -2.2 \\
Reported by: other health professional & 6.4 & 19 & 0.28 & -1.6 \\
Patient age: unknown & 11 & 28 & 0.34 & -1.5 \\
Reported by: lawyer & 0.01 & 23 & 0.37 & -1.4 \\
No. of adverse events per report: $3-5$ & 10 & 3 & 0.45 & -1.3 \\
Patient age: $20-29$ years & 6 & 55 & 0.57 & -0.9 \\
No. of drugs per report: 1 & 41 & 17 & 0.61 & -0.8 \\
Patient age: $50-59$ years & 11 & 9 & 0.59 & -0.6 \\
Patient age: $30-39$ years & 5 & 12 & 0.62 & -0.6 \\
Patient age: $40-49$ years & 8 & &
\end{tabular}

Fig. 2 Patient age distribution in Japanese reports (grey bars) compared with the expected age distribution in reports from the rest of the world (black lines)

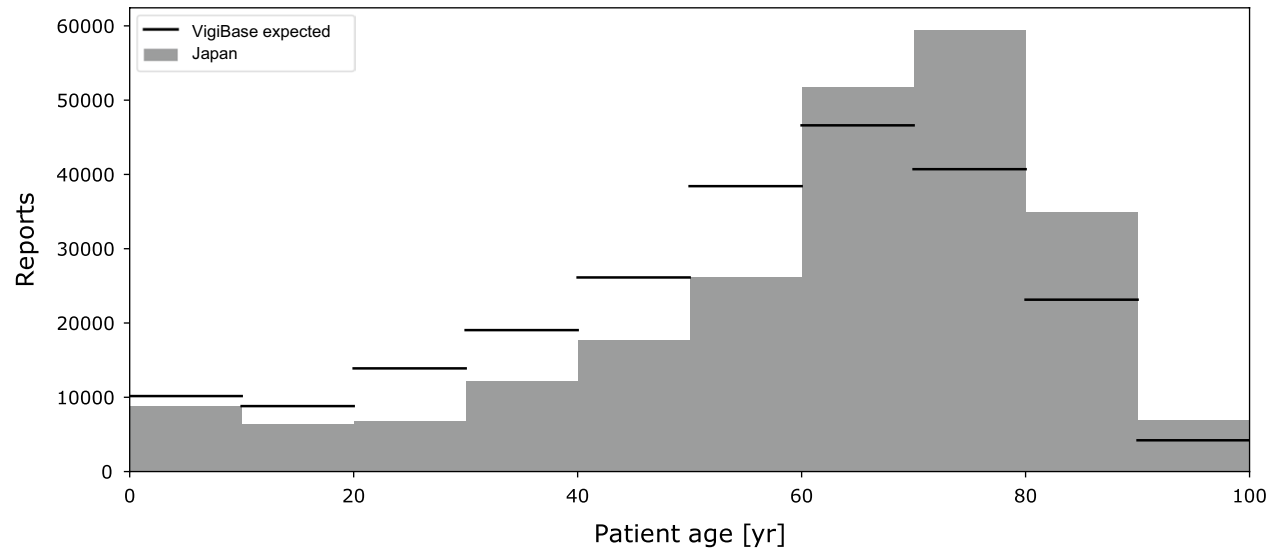

fewer adverse events but more drugs than do reports from the RoW.

Overall, reports from Japan tend to carry more information, as measured by the vigiGrade completeness score, as further illustrated in Fig. 3.

Table 5 presents the top ten adverse events identified as key features of reports from Japan with higher relative reporting rates than in reports from the RoW, as measured by the vigiPoint score. Table 6 presents the corresponding top ten adverse events with lower relative reporting rates in reports from Japan.

ILD is reported on $4 \%$ of the reports from Japan compared with on only $0.2 \%$ of the reports from the RoW. This reflects an asymmetry that has been described and studied previously $[5,6]$, and is analysed in more depth below. We also observed higher reporting rates of adverse events related to laboratory tests such as abnormal hepatic function and decreased platelet or neutrophil counts. There is 
Fig. 3 Distribution of vigiGrade completeness scores of Japanese reports (grey bars) compared with the expected distribution of reports from the rest of the world (black lines)

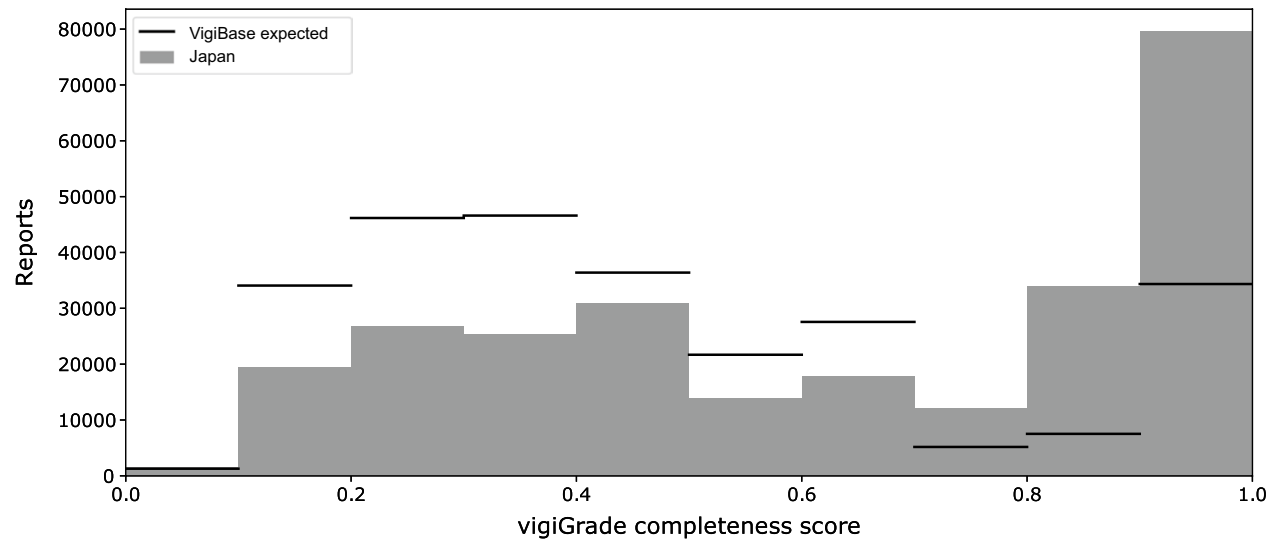

Table 5 Top ten adverse events identified as key features with higher relative reporting rates in reports from Japan

Table 6 Top ten adverse events identified as key features with lower relative reporting rates in reports from Japan

\begin{tabular}{lllll}
\hline MedDRA $^{\circledR}$ preferred terms & $\begin{array}{l}\text { Japan }(\%) \\
(n=261,052)\end{array}$ & $\begin{array}{l}\text { Rest of the world (\%) } \\
(n=2,522,856)\end{array}$ & Odds ratio & vigiPoint score \\
\hline Interstitial lung disease & 4.0 & 0.19 & 22 & 2.0 \\
Hepatic function abnormal & 2.2 & 0.078 & 29 & 1.5 \\
Platelet count decreased & 2.6 & 0.50 & 5.3 & 1.2 \\
Neutrophil count decreased & 1.7 & 0.23 & 7.4 & 1.1 \\
Drug eruption & 1.3 & 0.081 & 16 & 1.0 \\
Cerebral infarction & 1.3 & 0.14 & 9.9 & 1.0 \\
Renal impairment & 1.7 & 0.36 & 4.9 & 1.0 \\
Anaphylactic shock & 1.7 & 0.47 & 3.8 & 0.9 \\
Liver disorder & 1.1 & 0.15 & 6.9 & 0.8 \\
Erythema multiforme & 0.86 & 0.079 & 11 & 0.7 \\
\hline
\end{tabular}

\begin{tabular}{lllll}
\hline MedDRA $^{\circledR}$ preferred terms & $\begin{array}{l}\text { Japan }(\%) \\
(n=261,052)\end{array}$ & $\begin{array}{l}\text { Rest of the world (\%) } \\
(n=2,522,856)\end{array}$ & Odds ratio & vigiPoint score \\
\hline Death & 1.0 & 6.1 & 0.16 & -1.8 \\
Fatigue & 0.2 & 3.0 & 0.05 & -1.8 \\
Dyspnoea & 0.7 & 3.5 & 0.19 & -1.4 \\
Pain & 0.2 & 2.2 & 0.09 & -1.4 \\
Headache & 0.3 & 2.5 & 0.14 & -1.3 \\
Asthenia & 0.3 & 2.0 & 0.12 & -1.2 \\
Cerebrovascular accident & 0.05 & 1.5 & 0.04 & -1.2 \\
Myocardial infarction & 0.3 & 1.9 & 0.14 & -1.1 \\
Nausea & 0.9 & 3.1 & 0.27 & -1.1 \\
Off-label use & 0.01 & 1.2 & 0.01 & -1.1 \\
\hline
\end{tabular}

also higher reporting of cutaneous reactions such as drug eruption, erythema multiforme, and anaphylactic shock. These asymmetries are visible also for the corresponding MedDRA ${ }^{\circledR}$ HLTs platelet analyses $(2.6 \%$ vs. $0.6 \%)$, white blood cell analyses (3.2\% vs. 1.1\%), hepatic enzymes and function abnormalities ( $2.4 \%$ vs. $0.2 \%$ ), bullous conditions ( $2.4 \%$ vs. $0.9 \%)$, dermatitis ascribed to specific agent (2.4\% vs. $0.6 \%)$ and anaphylactic and anaphylactoid responses (3.1\% vs. $1.3 \%)$.
In contrast, most of the adverse events with lower relative reporting rates in reports from Japan are signs and symptoms that might be expected to have been co-reported with other adverse events on reports from the RoW, which list multiple adverse events more often than Japanese reports. The adverse events with lower reporting rates in Japan include fatigue, dyspnoea, pain, headache, asthenia and nausea. The higher reporting rate of cerebral infarction and lower reporting rate of cerebrovascular accident could reflect 
Table 7 Top ten suspected drug substances identified as key features of reports from Japan, which are more common

\begin{tabular}{lllll}
\hline WHODrug substance name & $\begin{array}{l}\text { Japan }(\%) \\
(n=261,052)\end{array}$ & $\begin{array}{l}\text { Rest of the world }(\%) \\
(n=2,522,856)\end{array}$ & Odds ratio & vigiPoint score \\
\hline Prednisolone & 2.3 & 0.3 & 7.6 & 1.3 \\
Methotrexate & 3.2 & 0.8 & 4.2 & 1.2 \\
Peginterferon alfa-2b & 1.2 & 0.1 & 13 & 1.0 \\
Gimeracil/oteracil/tegafur & 1.0 & 0.04 & 27 & 0.9 \\
Loxoprofen & 0.9 & 0.007 & 134 & 0.8 \\
Vildagliptin & 1.0 & 0.07 & 15 & 0.8 \\
Telaprevir & 1.3 & 0.3 & 5.2 & 0.8 \\
Tacrolimus & 1.8 & 0.5 & 3.4 & 0.8 \\
Red blood cells & 0.8 & 0.03 & 23 & 0.7 \\
Ribavirin & 1.7 & 0.6 & 2.7 & 0.7 \\
\hline
\end{tabular}

\begin{tabular}{lllll}
\hline WHODrug substance names & $\begin{array}{l}\text { Japan }(\%) \\
(n=261,052)\end{array}$ & $\begin{array}{l}\text { Rest of the } \\
\text { world }(\%) \\
(n=2,522,856)\end{array}$ & Odds ratio vigiPoint score \\
& 0 & 1.9 & 0 & -1.5 \\
\hline Rosiglitazone & 0.5 & 2.4 & 0.22 & -1.1 \\
Adalimumab & 0 & 1.2 & 0 & -1.1 \\
Blood substitutes and perfusion solutions & 0.8 & 2.9 & 0.28 & -1.1 \\
Lenalidomide & 0.007 & 1.2 & 0.01 & -1.0 \\
Calcium chloride; glucose; magnesium & & & & \\
$\quad$ chloride; sodium chloride; sodium & & & 0.25 & -1.0 \\
$\quad$ lactate & 0.6 & 2.4 & 0.03 & -0.9 \\
Etanercept & 0.04 & 1.1 & 0.02 & -0.9 \\
Levonorgestrel & 0.02 & 1.0 & 0.11 & -0.8 \\
Interferon beta-1a & 0.1 & 1.1 & 0.33 & -0.7 \\
Ambrisentan & 0.6 & 1.7 & & \\
Infliximab & & & & \\
\hline
\end{tabular}

Table 8 Top ten suspected drug substances identified as key features of reports from Japan, which are less common differences in coding preference, since these belong to the same MedDRA ${ }^{\circledR}$ HLT central nervous system haemorrhages and cerebrovascular accidents, for which the relative reporting rate is $3.7 \%$ from Japan and $3.1 \%$ from RoW. The lower relative reporting rate of myocardial infarction in reports from Japan on the other hand is reflected also for the MedDRA ${ }^{\circledR}$ HLT ischaemic coronary artery disorders, for which the relative reporting rate is $1.0 \%$ from Japan and $3.9 \%$ from the RoW.

Table 7 presents the top ten suspected drug substances identified as key features of reports from Japan with higher relative reporting rates than in reports from the RoW, as measured by the vigiPoint score. Table 8 presents the corresponding suspected drug substances with lower relative reporting rates in reports from Japan.

Prednisolone and methotrexate are the top two suspected drugs with higher relative reporting rates in Japan than in the RoW. The same holds also for their corresponding Anatomical Therapeutic Chemical (ATC) third-level groupings of Corticosteroids (2.4\% vs. $0.78 \%$ ) and Antimetabolites $(6.2 \%$ vs. $2.8 \%$ ), while Immunosuppressants are less reported in Japan than in the RoW (12\% vs. 16\%).

Three of the top ten substances, i.e. peginterferon alfa- $2 b$, ribavirin and telaprevir, reflect the standard triple therapy used to treat hepatitis $\mathrm{C}$ in Japan in the years before telaprevir was discontinued in 2017. Simeprevir, used with peginterferon alfa- $2 b$ and ribavirin for the same indication, was also identified as a key feature in our analysis, with a higher reporting rate in Japan $(0.7 \%$ vs. $0.1 \%)$.

The list also includes drugs that seem to be nearly exclusively used in Asia, such as loxoprofen, a non-steroidal anti-inflammatory drug, and the antineoplastic agent gimeracil/oteracil/tegafur. In all of VigiBase, $99 \%$ or more of the reports on these drugs come from countries in Asia. Both of these are developed by Japanese companies and the same is true for tacrolimus, which also has a higher relative reporting rate in Japan and is highlighted as a key feature. The patterns could also reflect differences in the extent of approved indications.

The higher relative reporting rates of red blood cells and the corresponding ATC third-level drug group Blood and 
related products in Japanese reports than in the RoW $(1.7 \%$ vs. $0.1 \%$ ) likely mirrors a difference in the regulatory classification of these products: in Japan, blood products are regulated as drugs and suspected adverse reactions associated with these are reported to the PMDA, which is not the case for many other countries.

While there is no overall difference in the reporting rates for the ATC third-level Blood glucose lowering drugs, excl. insulins ( $4.3 \%$ in both datasets), vildagliptin has a higher reporting rate for Japan while rosiglitazone, which is not on the Japanese market, naturally has a lower rate $(0 \%$ vs. $1.9 \%)$.

In our sensitivity analysis, the key features for lower reporting rates of rosiglitazone, blood substitutes and perfusion solutions, lenalidomide, ambrisentan and infliximab failed to reproduce except in the comparison against the Americas, and a closer review showed that it is the high reporting rates of these drugs in either the USA, Canada or both that deviate compared with for other countries in the programme. For lenalidomide, this is particularly striking: the reporting rate of lenalidomide in Japan is higher than that in each region except the Americas, and it is even highlighted as a key feature of high reporting in the vigiPoint comparisons with Africa and the Americas excluding the USA and Canada. Similarly, the reporting rate of ambrisen$\tan$ in Japan is the second highest of any individual country in the database, but because the reporting rate in the USA is so much higher, it was highlighted as a key feature of low reporting in our overall analysis.

With already noted exceptions, the key features presented earlier were robust to our sensitivity analyses and reproduced across the majority of our vigiPoint comparisons between Japan and specific regions of the world. When they did not, the reporting rate in Japan tended to deviate in the same direction as in the original analysis, but not enough to be highlighted as a key feature (e.g. the reporting rate from pharmacists, which was not highlighted as a key feature in comparison with Africa but was still higher in Japan than in Africa). Detailed results from the sensitivity analysis are available in the Electronic Supplementary Material.

\subsection{Key Features of Japanese Reports of Interstitial Lung Disease}

Our primary analysis identified a higher reporting rate of ILD in Japan than in the RoW. To assess whether this might reflect a coding preference, we evaluated all reports including an adverse event PT from the MedDRA ${ }^{\circledR}$ narrow SMQ for interstitial lung disease, ${ }^{3}$ and computed the relative proportion of such reports that included different MedDRA PTs

\footnotetext{
3 The SMQ also includes a number of MedDRA ${ }^{\circledR}$ LLTs, but these were not included in the analysis presented here.
}

Table 9 Top ten reported MedDRA ${ }^{\circledR}$ Preferred Terms within the Standardized MedDRA ${ }^{\circledR}$ Query for interstitial lung disease

\begin{tabular}{ll}
\hline MedDRA $^{\circledR}$ preferred term & $(\%)$ \\
\hline Japan & \\
Interstitial lung disease & 83 \\
Transfusion-related acute lung injury & 6.7 \\
Pneumonitis & 3.9 \\
Eosinophilic pneumonia & 2.4 \\
Idiopathic pulmonary fibrosis & 0.9 \\
Pulmonary fibrosis & 0.8 \\
Radiation pneumonitis & 0.5 \\
Eosinophilic granulomatosis with polyangiitis & 0.5 \\
Eosinophilic pneumonia acute & 0.3 \\
Pulmonary toxicity & 0.3 \\
Rest of the world & \\
Interstitial lung disease & 29 \\
Pneumonitis & 27 \\
Pulmonary fibrosis & 17 \\
Lung infiltration & 9.1 \\
Bronchiolitis & 4.1 \\
Pulmonary toxicity & 3.6 \\
Idiopathic pulmonary fibrosis & 2.7 \\
Eosinophilic pneumonia & 2.4 \\
Alveolitis allergic & 1.8 \\
Eosinophilic granulomatosis with polyangiitis & 1.2 \\
\hline
\end{tabular}

for reports from Japan and reports from RoW, respectively. The results are presented in Table 9.

As is clear from Table 9, the proportion of reports captured by the SMQ that includes the specific MedDRA ${ }^{\circledR}$ PT for ILD is much higher for Japan than for the RoW, and one might worry that cases of ILD in the RoW are coded as, for example, pneumonitis or pulmonary fibrosis. However, the overall relative reporting rate of the ILD narrow SMQ in Japan is $4.8 \%$ versus $0.6 \%$ in the RoW. Based on this, there seems to be a coding preference that contributes to the difference in the relative reporting rate of ILD between Japan and the RoW, but this alone does not seem to explain the difference. It also cannot be explained by the higher reporting of blood products in Japan, since they account for only $6.8 \%$ of all Japanese reports on the ILD SMQ (results not shown here). We therefore base the subsequent vigiPoint analysis on the MedDRA ${ }^{\circledR}$ PT for ILD to avoid confounding by the different distribution of MedDRA ${ }^{\circledR}$ PTs for the two subsets.

Overall, there are 10,432 reports on ILD from Japan and 4782 from the RoW. Table 10 presents key features with higher relative reporting rates in the subset of ILD reports from Japan as compared with reports on ILD from the RoW and reports on other adverse events from Japan. Table 11 presents key features with lower relative reporting rates in the subset of Japanese reports on ILD. 
Table 10 Key features of interstitial lung disease reports from Japan, which are more common

\begin{tabular}{lllll}
\hline Feature & $\begin{array}{l}\text { ILD in Japan }(\%) \\
(n=10,432)\end{array}$ & $\begin{array}{l}\text { Other adverse events in Japan } \\
(\%)(n=261,052)\end{array}$ & $\begin{array}{l}\text { ILD in the rest of the world vigiPoint score } \\
(\%)(n=4782)\end{array}$ \\
\hline Completeness: high & 63 & 52 & 35 & 0.6 \\
No. of reported adverse events: one & 79 & 72 & 50 & 0.5 \\
Reported by: physician & 88 & 83 & 66 & 0.5 \\
\hline
\end{tabular}

ILD interstitial lung disease

Table 11 Key features of interstitial lung disease reports from Japan, which are less common

\begin{tabular}{lllll}
\hline Feature & $\begin{array}{l}\text { ILD in } \\
\text { Japan }(\%) \\
(n=10,432)\end{array}$ & $\begin{array}{l}\text { Other adverse events in } \\
\text { Japan }(\%)(n=261,052)\end{array}$ & $\begin{array}{l}\text { ILD in the rest of the } \\
\text { world }(\%)(n=4782)\end{array}$ & vigiPoint score \\
\hline $\begin{array}{l}\text { Reported by: consumer/ } \\
\text { non-health profes- } \\
\text { sional }\end{array}$ & 3.9 & 9.4 & 14 & -1.0 \\
Patient age: $20-29$ years & 0.5 & 3.1 & 1.9 & -0.6 \\
$\begin{array}{l}\text { Patient age: } 30-39 \text { years } \\
\text { Patient age: } 40-49 \text { years }\end{array}$ & 1.3 & 5.4 & 3.2 & -0.6 \\
\hline
\end{tabular}

$I L D$ interstitial lung disease

\begin{tabular}{lllll}
\hline $\begin{array}{l}\text { WHODrug } \\
\text { substance name }\end{array}$ & $\begin{array}{l}\text { ILD in Japan }(\%) \\
(n=10,432)\end{array}$ & $\begin{array}{l}\text { Other adverse events in } \\
\text { Japan }(\%)(n=261,052)\end{array}$ & $\begin{array}{l}\text { ILD in the rest of the } \\
\text { world }(\%)(n=4782)\end{array}$ & vigiPoint score \\
\hline Cetuximab & 2.6 & 0.4 & 0.5 & 1.0 \\
Temsirolimus & 2.1 & 0.1 & 0.4 & 0.9 \\
Nivolumab & 5.3 & 0.8 & 2.1 & 0.9 \\
Gefitinib & 1.9 & 0.2 & 0.7 & 0.6 \\
Irinotecan & 1.4 & 0.4 & 0.2 & 0.5 \\
Panitumumab & 1.1 & 0.2 & 0.2 & 0.5 \\
Paclitaxel & 3.5 & 0.8 & 1.9 & 0.5 \\
\hline
\end{tabular}

ILD interstitial lung disease

Table 12 Suspected drug substances identified as key features with higher relative reporting rates in reports on interstitial lung disease from Japan than in reports on interstitial lung disease from the rest of the world and in reports on other adverse events from Japan
Table 12 presents the suspected drugs highlighted as key features of reports on ILD in Japan. All these substances have ILD listed as an ADR in their European summary of product characteristics (SPC) and also in the Japanese label $[7,8]$. As a complement, Table 13 displays the most common drug substances on Japanese reports with ILD overall, including seven that were not highlighted as key features because they are commonly reported with ILD also in the RoW.

\section{Discussion}

Our study has identified a number of ways in which the reporting of serious suspected ADRs differs between Japan and other countries in the WHO Programme for International Drug Monitoring. Further consideration of these differences point to a variety of possible explanations. 
Table 13 Suspected drug substances with the highest relative reporting rates in reports on interstitial lung disease in Japan. Drug substances highlighted as key features are in italics

\begin{tabular}{lllll}
\hline $\begin{array}{l}\text { WHODrug } \\
\text { substance names }\end{array}$ & $\begin{array}{l}\text { ILD in Japan }(\%) \\
(n=10,432)\end{array}$ & $\begin{array}{l}\text { Other adverse events in } \\
\text { Japan }(\%)(n=261,052)\end{array}$ & $\begin{array}{l}\text { ILD in the rest of the } \\
\text { world }(\%)(n=4782)\end{array}$ & vigiPoint score \\
\hline Everolimus & 7.2 & 0.8 & 4.9 & 0.4 \\
Methotrexate & 6.6 & 3.1 & 7.5 & 0 \\
Nivolumab & 5.3 & 0.8 & 2.1 & 0.9 \\
Paclitaxel & 3.5 & 0.8 & 1.9 & 0.5 \\
Gemcitabine & 3.3 & 0.5 & 2.5 & 0.1 \\
Docetaxel & 3.2 & 0.8 & 2.2 & 0.2 \\
Cetuximab & 2.6 & 0.4 & 0.5 & 1.0 \\
Erlotinib & 2.4 & 0.3 & 1.9 & 0.05 \\
Amiodarone & 2.4 & 0.2 & 12.3 & 0 \\
Fluorouracil & 2.3 & 1.0 & 1.2 & 0.4 \\
\hline
\end{tabular}

$I L D$ interstitial lung disease
A small number of the identified differences may reflect possible pharmaco-ethnic vulnerabilities or strengths. The lower reporting rate of myocardial infarction and other ischaemic coronary artery disorders mirrors an overall lower rate of cardiovascular disease in Japan [9]. In contrast, we have not been able to find an explanation for the higher reporting rates of erythema multiforme and drug eruption as suspected ADRs in Japan, so this may merit further evaluation. We do observe that among the drugs with higher reporting rates of these severe skin reactions in Japan are lamotrigine, carbamazepine and celecoxib (results not shown here). For carbamazepine, there exists a known association between the HLA-A*3101 allele and hypersensitivity reactions. This association is included in carbamazepine labelling in Japan, the USA and the European Union (EU): "Retrospective case-control studies in patients of European, Korean, and Japanese ancestry have found a moderate association between the risk of developing hypersensitivity reactions and the presence of HLAA*3101, an inherited allelic variant of the HLA-A gene, in patients using carbamazepine." [10]. For lamotrigine and celecoxib, we have not been able to identify any such evidence so they may require further evaluation for possible unknown genetic susceptibility in the Japanese or alternative explanations to the higher adverse event reporting rates in Japan. It may be relevant that lamotrigine and carbamazepine (but not celecoxib) were included in a PMDA alert related to druginduced serious skin disorders issued in April 2012 [11]. It should be acknowledged that while spontaneous reports may possibly help generate hypotheses of pharmaco-ethnic effects, they do not support conclusive inference, since observed differences in reporting rates may be driven not by differences in occurrence but by differences in diagnosis, reporting and coding of adverse events.

Some of the observed patterns are likely to reflect differences in drug use. The higher reporting rate of anaphylactic shock is associated with higher reporting rates in Japan of anaphylactic reactions related to blood substitutes and perfusion solutions (ATC B05) and contrast media (V08) (results not shown here). As noted earlier, not all countries share reports on adverse events related to blood products into VigiBase and Japan is an Organisation for Economic Co-operation and Development (OECD) top user of computed tomography (CT) scans per capita [12]. Similarly, the higher reporting rates of adverse events related to low platelet and white blood cell counts could at least partly be explained by the higher reporting of antineoplastic agents, which can lead to bone marrow depression; 25\% of the reports from Japan include an antineoplastic agent compared with $15 \%$ for the RoW.

Differences may also reflect variations in reporting culture. The higher reporting of laboratory test results such as low platelet counts, etc. might be a result of active Marketing Authorisation Holder follow-up of the adverse events reported by healthcare providers.

Some differences in reporting can probably be explained by coding preferences, as was noted for cerebral infarction and cerebrovascular accident. For ILD, we observed some evidence of coding preference (greater tendency to report the PT interstitial lung disease in Japan) as has also been highlighted in earlier research [6], but not enough to fully explain the higher reporting rate of this and related adverse events in Japan. The in-depth analysis of ILD reports in Japan versus in the RoW highlighted higher reporting rates for antineoplastic drugs for which ILD is a labelled ADR. This could point to some possible explanations for the overall higher rate of ILD as an adverse event in Japan, but our analyses have not been able to fully explain this asymmetry.

A greater proportion of Japanese reports relate to patients of age 70 years and above, and a lower proportion to younger adults (20-59 years of age), as might be expected from the overall demographics of the country. Initially, we hypothesized that the higher reporting rates of adverse events such as renal failure and impairment and liver disorder might be explained by the older Japanese population, but a subsequent 
sensitivity analysis disproved this: these two adverse events remained key features of Japanese reports in a vigiPoint analysis restricted to patients in the higher age range (results not shown here).

A number of the highlighted reporting patterns may be important to be aware of in subsequent analyses. The higher proportions of reports submitted by physicians or pharmacists and absence of direct patient reports will likely impact the types of adverse event terms that tend to be used. An explanation for the lower median number of adverse event terms on Japanese reports may be close adherence to the MedDRA ${ }^{\circledR}$ guideline to select a term for the diagnosis only, and not any co-reported signs and symptoms. This may impact the possibility to successfully apply novel methods for data analysis such as adverse event cluster analysis that rely on the co-reporting of multiple adverse event terms to characterise an observed adverse event [13]. Similarly, the lower proportion of reports from Japan with death listed as an adverse event term (1\% vs. $6 \%$ ) likely reflects adherence to the MedDRA ${ }^{\circledR}$ recommendation to code this information in other fields, since our analysis of fatal cases using several reporting elements found similar proportions in Japan and the RoW (12\% vs. $15 \%)$.

The higher completeness of information is a strength of the Japanese reporting system that should be maintained and reinforced. It offers opportunities to explore other dimensions of reported adverse events, such as dosages and time to onset. The higher completeness of information may also help explain the observed greater number of listed drugs per report in Japan if it means that concomitant medications are more completely noted, but a hypothesized contributing factor could also be a higher degree of polypharmacy related to the older population.

It should be acknowledged that the analyses presented here are based on reports between 1 January 2013 and 2 January 2018, and that the nature of reporting will change with time. For example, direct patient reporting became possible in Japan in March 2019 and the proportion of reports in the Japanese subset with consumer as one of the reporter qualifications is likely to increase in the coming years. Similarly, it is important to be aware of the large influence of US reports in the comparator ( $51 \%$ of the reports in the RoW data). This reflects the current composition of VigiBase, but also means that some observed differences can be driven by US reporting patterns that may not be reflected in most other countries.

vigiPoint is focused on the main effects and may miss interactions. As a hypothetical example, if Japanese consumer reports had had lower completeness than consumer reports from the RoW, this may not have been detected in our main analysis where consumer reports make up a small proportion of reports from Japan. With the exception of the in-depth analysis of ILD reports, reporting patterns related to drug-event pairs are also out of the scope of our analysis presented here. Consequently, our analysis will by design ignore patterns such as the fact that more than $20 \%$ of reports on methotrexate from Japan are for lymphoproliferative disorders compared with around $2 \%$ for reports on methotrexate from other countries (results not shown here).

vigiPoint may be subject to confounding. As an example, the lower average number of adverse event terms per report (and higher average number of drugs) complicate any comparisons of relative reporting rates of drugs and adverse events between Japan and the RoW; in theory, every adverse event could have a higher reporting rate in the RoW and every drug a higher reporting rate in Japan.

Such possibilities need to be carefully considered, and it is important that vigiPoint results be seen as starting points for subsequent exploratory analyses and review, rather than as final answers in their own right. Nested vigiPoint analyses offer one avenue for follow-on analysis, as illustrated by our analysis of ILD reports in this study.

Exclusion criteria are an additional source of possible erroneous conclusions and missed insights. They may hide important features related to the criteria themselves. For example, a greater proportion of Japanese reports in VigiBase are serious in nature than is the case for reports from the RoW, but this is not visible in the vigiPoint analysis because of the initial exclusion of non-serious reports. This is important to be aware of if subsequent analyses apply different exclusion criteria, since findings of the analyses presented here may not generalise to such settings.

The standard implementation of vigiPoint as used here sets quite a high threshold on the magnitude of the difference in reporting rates as well as on its absolute value for features to be highlighted. As a result, our analysis did not highlight some potentially interesting patterns such as the more balanced patient sex distribution in Japan than in the RoW where there are significantly more reports for women, because the magnitude of the difference between the observed and expected was not large enough.

A more subtle challenge is the choice of comparator(s) in nested vigiPoint analysis. Our nested analysis of ILD in Japan used a dual comparator of reports on other adverse events in Japan and ILD reports from the RoW. As a result, to be identified as a key feature, the reporting rate of a covariate had to exceed both that for ILD in the RoW and that for other adverse events in Japan. This makes sense, for example, in looking for drugs more commonly reported with ILD in Japan, where we want to avoid highlighting drugs that are just generally more common in Japan but not specifically so for ILD. On the other hand, it is less well-suited to highlight drugs less commonly reported with ILD in Japan versus in the RoW, because to be highlighted, the drugs then also must have a lower reporting rate with ILD than in Japan overall, which is unlikely if they are associated with ILD.

Finally, as with any analysis of spontaneous reports, we cannot automatically disentangle different prevalences of 
drugs and adverse events in the population from differential reporting rates. Is this drug with a high reporting rate used more and/or do we see more adverse events for it and/or are they reported to a greater extent?

\section{Conclusions}

Japanese reports make up a substantial proportion of VigiBase, the WHO global database of individual case safety reports, and exhibit several key features that are important to be aware of. Comparisons between reports from one country and reports from the RoW rely on countries to share their data with one another and allows for analyses that can highlight possible pharmaco-ethnic vulnerabilities as well as features of a data subset that provide important perspectives on subsequent analyses. The vigiPoint methodology offered efficient, reproducible and transparent exploratory analysis for the purpose of this study that should be taken as starting point for further careful review and evaluation.

Acknowledgements The views expressed in this article are the personal views of the authors and may not be understood or quoted as being made on behalf of or reflecting the position of the Pharmaceuticals and Medical Devices Agency (PMDA). The authors are indebted to the national centres that make up the World Health Organization (WHO) Programme for International Drug Monitoring and contribute reports to VigiBase. However, the opinions and conclusions of this study are not necessarily those of the various centres nor of the WHO.

\section{Compliance with Ethical Standards}

Funding No funding was received for this study.

Conflict of interest Rika Wakao, Henric Taavola, Lovisa Sandberg, Eiko Iwasa, Saori Soejima, Rebecca Chandler and G. Niklas Norén have no conflicts of interest that are directly relevant to the content of this study.

Open Access This article is distributed under the terms of the Creative Commons Attribution-NonCommercial 4.0 International License (http://creativecommons.org/licenses/by-nc/4.0/), which permits any noncommercial use, distribution, and reproduction in any medium, provided you give appropriate credit to the original author(s) and the source, provide a link to the Creative Commons license, and indicate if changes were made.

\section{References}

1. Norén GN, Orre R, Bate A, Edwards IR. Duplicate detection in adverse drug reaction surveillance. Data Min Knowl Discov. 2007;14(3):305-28. https://doi.org/10.1007/s10618-006-0052-8.

2. Juhlin K, Star K, Norén GN. A method for data-driven exploration to pinpoint key features in medical data and facilitate expert review. Pharmacoepidemiol Drug Saf. 2017;26(10):1256-65. https://doi.org/10.1002/pds.4285.

3. Norén GN, Hopstadius J, Bate A. Shrinkage observed-toexpected ratios for robust and transparent large-scale pattern discovery. Stat Methods Med Res. 2013;22(1):57-69. https://doi. org/10.1177/0962280211403604.

4. Bergvall T, Norén GN, Lindquist M. vigiGrade: a tool to identify well-documented individual case reports and highlight systematic data quality issues. Drug Saf. 2014;37(1):65-77. https://doi. org/10.1007/s40264-013-0131-x.

5. Koo LC, Clark JA, Quesenberry CP, Higenbottam T, Nyberg F, Wolf MK, et al. National differences in reporting 'pneumonia' and 'pneumonia interstitial': an analysis of the WHO International Drug Monitoring Database on 15 drugs in nine countries for seven pulmonary conditions. Pharmacoepidemiol Drug Saf. 2005;14(11):775-87. https://doi.org/10.1002/pds.1071.

6. Pinheiro L, Blake K, Januskiene J, Yue Q-Y, Arlett P. Geographical variation in reporting Interstitial Lung Disease as an adverse drug reaction: findings from an European Medicines Agency analysis of reports in EudraVigilance. Pharmacoepidemiol Drug Saf. 2016;25(6):705-12. https://doi.org/10.1002/pds.3998.

7. European Medicines Agency. Medicines. 2018. https://www.ema. europa.eu/en/medicines. Accessed 29 Nov 2018.

8. Pharmaceuticals and Medical Devices Agency. PMDA labels [in Japanese]. 2019. https://www.pmda.go.jp/PmdaSearch/iyakuSearc $\mathrm{h} /$. Accessed 6 Mar 2019.

9. OECD. The burden of cardiovascular disease and diabetes. In: Cardiovascular disease and diabetes: policies for better health and quality of care. Paris: OECD Publishing; 2015. p. 31-51. https:// doi.org/10.1787/9789264233010-5-en.

10. US FDA. Table of pharmacogenomic biomarkers in drug labeling. Last updated 06/2019. https://www.fda.gov/media/124784/downl oad. Accessed 5 Aug 2019.

11. PMDA. Early detection of drug-induced serious skin disorders. In: PMDA alert for proper use of drugs. Tokyo: PMDA; 2012. https ://www.pmda.go.jp/files/000153436.pdf. Accessed 2 Dec 2018.

12. OECD. Computed tomography (CT) exams (indicator). 2018. https://doi.org/10.1787/3c994537-en. Accessed 27 Nov 2018.

13. Chandler RE, Juhlin K, Fransson J, Caster O, Edwards IR, Norén GN. Current safety concerns with human papillomavirus vaccine: a cluster analysis of reports in VigiBase ${ }^{\circledR}$. Drug Saf. 2017;40(1):81-90. https://doi.org/10.1007/s40264-016-0456-3.

\section{Affiliations}

\section{Rika Wakao ${ }^{1}$ Henric Taavola ${ }^{2}$ [ - Lovisa Sandberg ${ }^{2}$ - Eiko Iwasa ${ }^{1} \cdot$ Saori Soejima ${ }^{1} \cdot$ Rebecca Chandler $^{2}$. G. Niklas Norén ${ }^{2}(\mathbb{C}$}

Henric Taavola

henric.taavola@who-umc.org
2 Uppsala Monitoring Centre, Box 1051, 75140 Uppsala, Sweden

1 Pharmaceuticals and Medical Devices Agency, Tokyo, Japan 